\title{
Formal Solution for the Fields within a Beam-Bug Calibrator
}

\author{
T.J. Fessenden
}

July 13, 1998

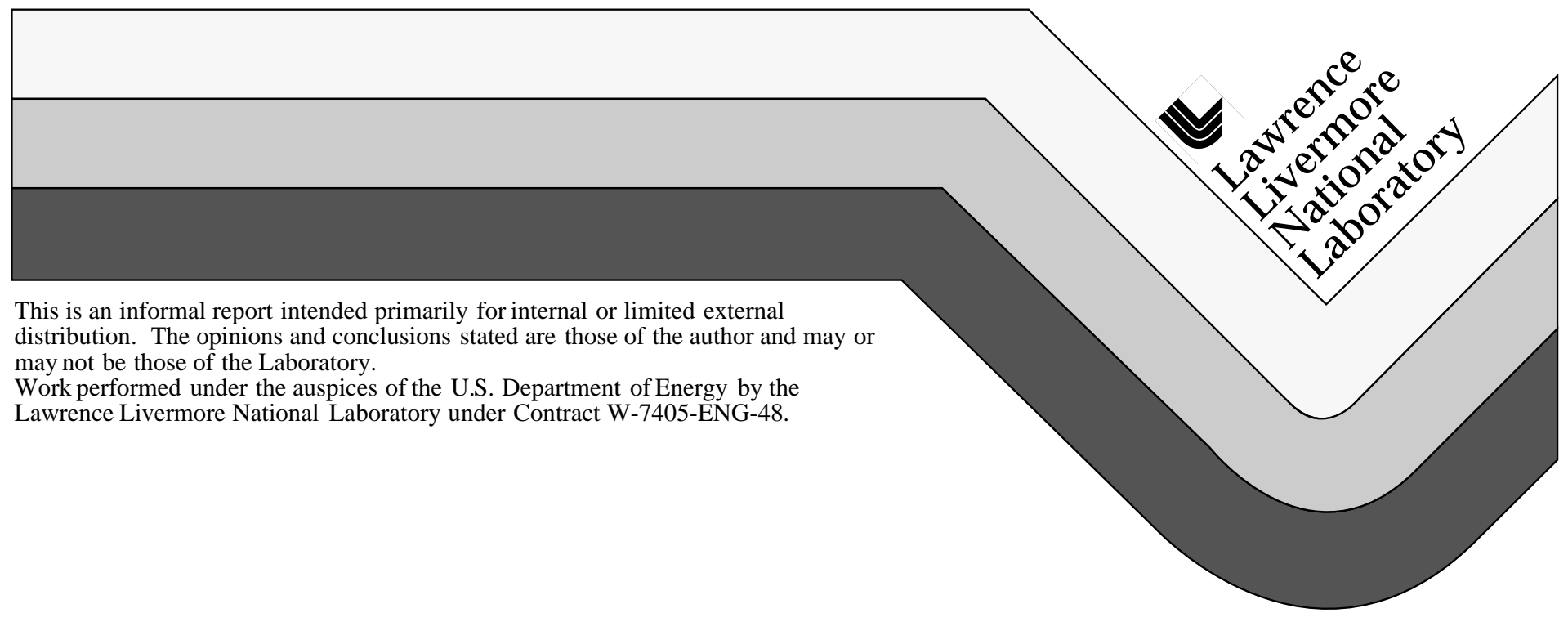




\section{DISCLAIMER}

This document was prepared as an account of work sponsored by an agency of the United States Government. Neither the United States Government nor the University of California nor any of their employees, makes any warranty, express or implied, or assumes any legal liability or responsibility for the accuracy, completeness, or usefulness of any information, apparatus, product, or process disclosed, or represents that its use would not infringe privately owned rights. Reference herein to any specific commercial product, process, or service by trade name, trademark, manufacturer, or otherwise, does not necessarily constitute or imply its endorsement, recommendation, or favoring by the United States Government or the University of California. The views and opinions of authors expressed herein do not necessarily state or reflect those of the United States Government or the University of California, and shall not be used for advertising or product endorsement purposes.

This report has been reproduced directly from the best available copy.

Available to DOE and DOE contractors from the Office of Scientific and Technical Information P.O. Box 62, Oak Ridge, TN 37831

Prices available from (423) 576-8401

Available to the public from the National Technical Information Service

U.S. Department of Commerce 5285 Port Royal Rd., Springfield, VA 22161 


\title{
Formal solution for the fields within a beam-bug calibrator
}

\author{
T.J. Fessenden \\ $7 / 13 / 98$
}

For some time I was bothered by the fact that measurements of offsets in the various bug calibration setups never agreed with the simple formulae (2) used for determining electron beam position in the Livermore induction linacs and transport systems. About 1983 I realized that the discrepancy arises from the way the bug calibrator simulates an electron beam in a conducting pipe. At that time I solved the problem using the method presented here. Unfortunately, I did not write it up at that time. After considerable effort, I was able to repeat the calculation. Since I have little confidence that after a few years I could ever do it again, I felt obliged to write it up in some detail.

Our beam bug calibrator consists of two conducting cylinders, nominally concentric, that simulate the electron beam within a drift tube. The radii of the larger cylinder is 2.3 times that of the smaller giving an electrical impedance of $50 \mathrm{Ohms}$ to the coaxial combination. To simulate a beam off-axis within a drift tube, the inner tube is moved relative to the outer tube. This only approximately simulates the motion of a beam because the surface current on the inner tube redistributes in response to the translation. Fortunately, the fields of the translated inner cylinder can be found exactly using complex variable theory (1).

\section{$\square$ Theory}

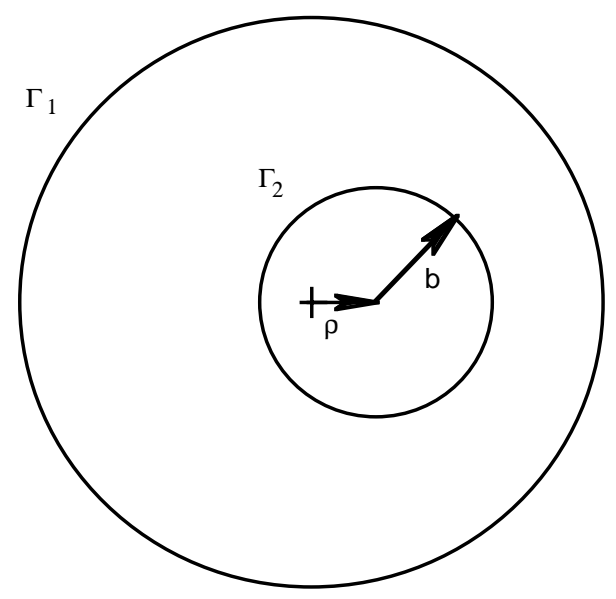

Fig 1. Sketch of a cylinder $\Gamma 2$ of radius $b$ offset by $\rho$ from the axis of $\Gamma 1$

Two circular cylinders can be mathematically described in the complex z plane by: $\Gamma 1:|z|=1$ and $\Gamma 2:|z-\rho|=b$.

Here $\mathrm{z}$ is the complex variable $\mathrm{x}+$ iy and $\rho$ is the normalized offset of the axis of the smaller cylinder $(\Gamma 2)$ of normalized radius $b$. The normalizations are taken to the radius of the larger cylinder. If the coordinates are chosen in the direction of the offset, the parameters $b$ and $\rho$ are real.

Consider the biquadratic mapping 


$$
(\mathbf{z}-\alpha) /(\mathbf{z}-\beta)=\kappa
$$

Here $\alpha, \beta$, and $\mathrm{k}$ are real. This mapping has the property that circles map to circles or lines (a circle of infinite radius). We define $\alpha$ and $\beta$ as inverse points of both $\Gamma 1$ and $\Gamma 2$. That is they satisfy the relations

$$
\begin{aligned}
& \alpha * \beta=1 \\
& (\alpha-\rho) *(\beta-\rho)=\beta^{2}
\end{aligned}
$$

Solving for $\alpha, \beta$ yields:

$$
\begin{aligned}
& \alpha\left[b_{-}, \rho \_\right]:=\left(1-b^{\wedge} 2+\rho^{\wedge} 2-S q r t\left[\left(1-b^{\wedge} 2+\rho^{\wedge} 2\right)^{\wedge} 2-4 \rho^{\wedge} 2\right]\right) /(2 \rho) \\
& \beta e\left[b \_, \rho\right]:=1 / \alpha[b, \rho]
\end{aligned}
$$

With these definitions the solution for the complex potential between the displaced cylinders as shown in Ref. 1 is given by

$$
\Psi=\mathrm{K} \ln (\mathbf{z}-\alpha) /(\mathbf{z}-\beta)
$$

The electrical potential is the real part of this complex potential and is given by

$$
\vartheta=\operatorname{Re} \Psi=K|\ln (\mathbf{z}-\alpha) /(z-\beta)|
$$

Note the absolute value signs. The physics of the problem determine the value of $\mathrm{K}$.

The electric field around the circumference of $\Gamma 1$ is given by

$$
\mathrm{E}=-\partial \vartheta / \partial \rho \text { evaluated at }|\mathbf{z}|=1
$$

This is then the surface charge distribution on the inside of $\Gamma 1$ or the surface current distribution in the analogous problem of interest. Evaluating this function taking proper care of the absolute values yields the result for the current density apart from the constant $\mathrm{K}$ around the outer cylinder as:

$$
\begin{aligned}
k\left[b \_, \rho_{-}, \phi\right]:= & (1-\alpha[b, \rho] \operatorname{Cos}[\phi]) /\left(1+\alpha[b, \rho]^{\wedge} 2-2 \alpha[b, \rho] \operatorname{Cos}[\phi]\right)- \\
& (1-\beta \varepsilon[b, \rho] \operatorname{Cos}[\phi]) /\left(1+\beta \varepsilon[b, \rho]^{\wedge} 2-2 \beta \varepsilon[b, \rho] \operatorname{Cos}[\phi]\right)
\end{aligned}
$$

The constant $\mathrm{K}$ is found by integrating $\mathrm{k}$ around the circumference of the outer cylinder and setting the result equal to the current I. We find

$$
\mathrm{K}=\mathrm{I} /(2 \pi \mathbf{r})
$$

where $r$ is the radius of the outer cylinder. This constant can also be represented in terms of the voltage Vc generated across the bug with no offset as

$$
K=\mathbf{V} / \mathbf{R}
$$

To connect with previous work we note that if the radius of the small cylinder $b$ is set to zero we find $\alpha=\rho, \beta=1 / \rho$ and the result presented in Ref (2).

$$
k[\rho, \phi]=\left(1-\rho^{\wedge} 2\right) /\left(1+\rho^{\wedge} 2-2 \rho \operatorname{Cos}[\phi]\right)
$$

Defining a position function pos as the voltage difference generated by an offset divided by the centered voltage $\mathrm{Vc}$ we find: 


$$
\operatorname{pos}\left[\mathbf{b}, r \_\right]:=(k[b, \rho, 0]-k[b, \rho, P i])
$$

Performing some algebra yields a simpler form for this position function:

$$
\text { pos }\left[b \_, \rho\right]:=4 \alpha[b, \rho] /\left(1-\alpha[b, \rho]^{\wedge} 2\right)
$$

\section{Some Results}

Let us plot a few of these functions for various cases of interest. First consider the above function that represents a displacement along the line of the pickoffs.

p1 = Plot[pos $[.4, \rho],\{\rho, 0.001, .5\}$, GridLines->Automatic $]$

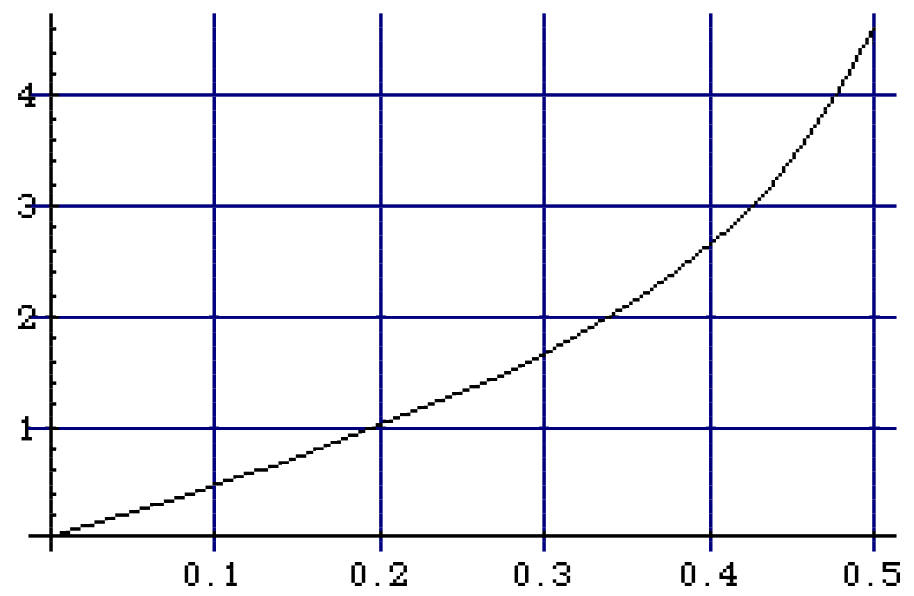

Fig 2. Theoretical plot of the position function versus the normalized radius. The ordinate is $(V+-V-) / V c$ where $V+$ is the voltage toward the offset, $V$ - is the voltage away from the offset, and $V c$ is the voltage with no offset.

For small offsets $\rho$ this position function is given by :

$$
\operatorname{pos}\left[b_{-}, \rho_{-}\right]:=4 \rho /\left(1-b^{\wedge} 2\right)
$$

Although these are mostly of academic interest, it is interesting to look at a number of different cases to fill out the analysis. Let us look at offsets at 30, 45, and 60 degrees as well as at zero degrees to the direction of the pickoffs. For these cases we have a position functions given by

$$
\begin{aligned}
& \operatorname{pos} 30\left[b_{-}, \rho\right]:=k[b, \rho, P i / 6]-k[b, \rho, 7 \mathrm{Pi} / 6] \\
& \operatorname{pos} 45\left[b_{-}, \rho\right]:=k[b, \rho, 0.25 \mathrm{Pi}]-k[b, \rho,-0.75 \mathrm{Pi}] \\
& \operatorname{pos} 60\left[b \_, \rho\right]:=k[b, \rho, P i / 3]-k[b, \rho, 4 \mathrm{Pi} / 3]
\end{aligned}
$$

Defining these plots

$$
\begin{aligned}
& \text { p2 }=\operatorname{Plot}[\operatorname{pos} 30[.4, \rho],\{\rho, 0.001,0.5\}] \\
& \text { p3 }=\operatorname{Plot}[\operatorname{pos} 45[.4, \rho],\{\rho, 0.001,0.5\}] \\
& \text { p4 }=\operatorname{Plot}[\operatorname{pos} 60[.4, \rho],\{\rho, 0.001,0.5\}]
\end{aligned}
$$

Finally, plotting these all on the same scale gives: 
Show $[p 1, p 2, p 3, p 4]$

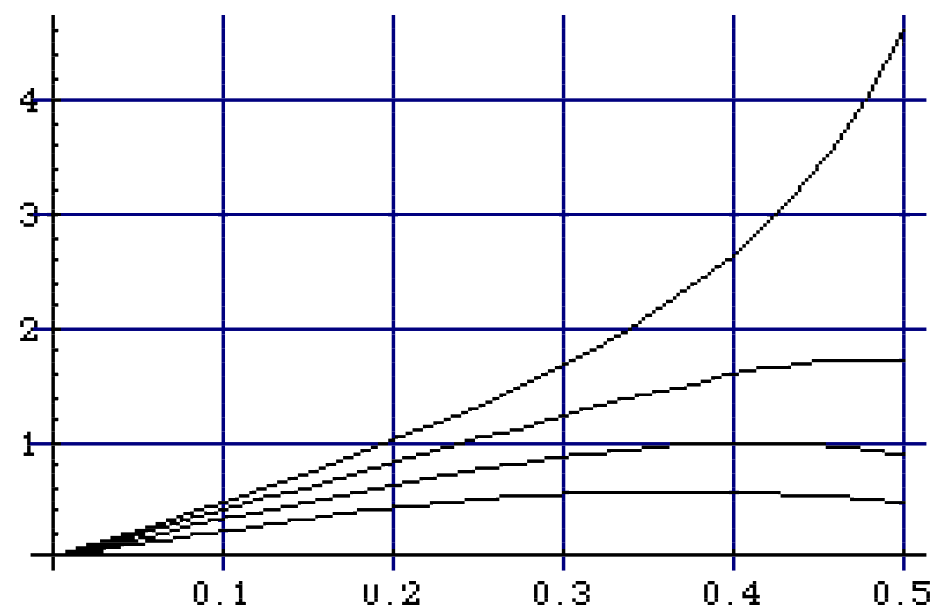

Fig 3. Plots of the position function at angles of 0, 30, 45, and 60 degrees to the direction of the pickoffs for normalized offsets of 0 to 0.5 .

As a last case consider the current function formed by pickoffs at the four cardinal points defined as:

$\operatorname{cur}\left[b_{-}, \rho_{-}, \phi\right]:=(k[b, \rho, \phi]+k[b, \rho, \phi+P i / 2]+k[b, \rho, \phi+P i]+k[b, \rho, \phi+3$ $\mathrm{Pi} / 2]) / 4$ p5 = Plot $[\operatorname{cur}[.4, \rho, 0],\{\rho, .001, .5\}$, GridLines->Automatic $]$

Similarly, at 45 degrees from these points we have p6 = Plot[cur[.4, $\rho, \Pi / / 4],\{\rho, .001, .5\}$, GridLines->Automatic $]$ Plotting these two curves gives.

\section{Show[p5,p6, PlotRange $->\{\{0, .5\},\{.8,1.2\}\}]$}

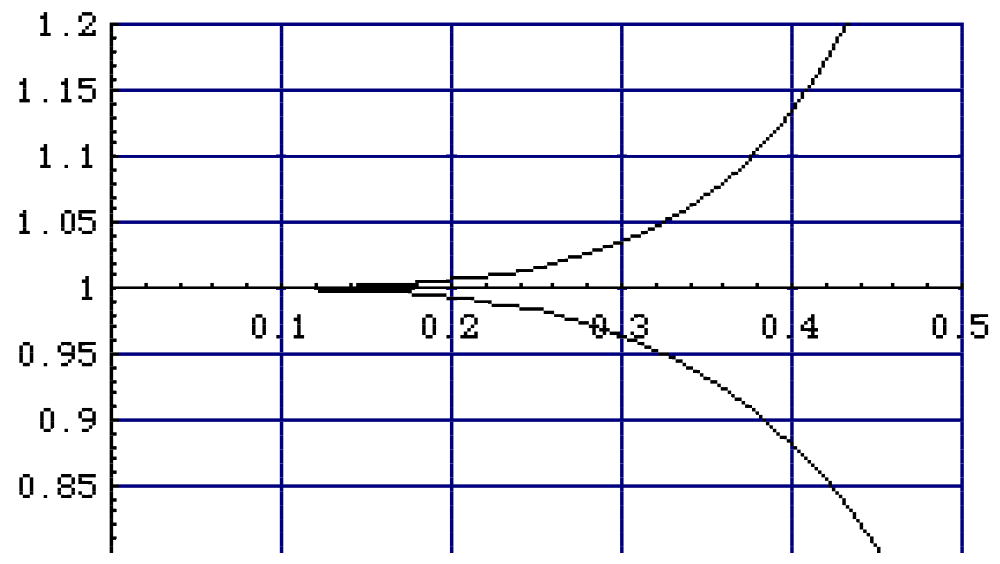

Fig. 4 Plots of the current function for offsets toward one of the pickoffs and half way between pickoffs for the case of a $50 \mathrm{Ohm}$ calibrator. 


\section{$\square$ Comparison with Experiment}

John Clark and I performed an experiment to check this theory. A 200 ns pulse was sent down a coaxial line containing a standard beam bug. We displaced the center conductor of the line with respect to the outer conductor and recorded the difference in pick up voltages from each side of the bug as a function of the displacement. The difference voltage was normalized to the on-axis voltage and the displacement was normalized to the radius of the outer conductor. Because of mechanical limitations, the displacement was limited to 15 $\mathrm{mm}$ or a normalized displacement of 0.227 . These data are plotted below.

\section{data = ReadList["data", \{Number, Number\}];}

\section{ListPlot[data];}

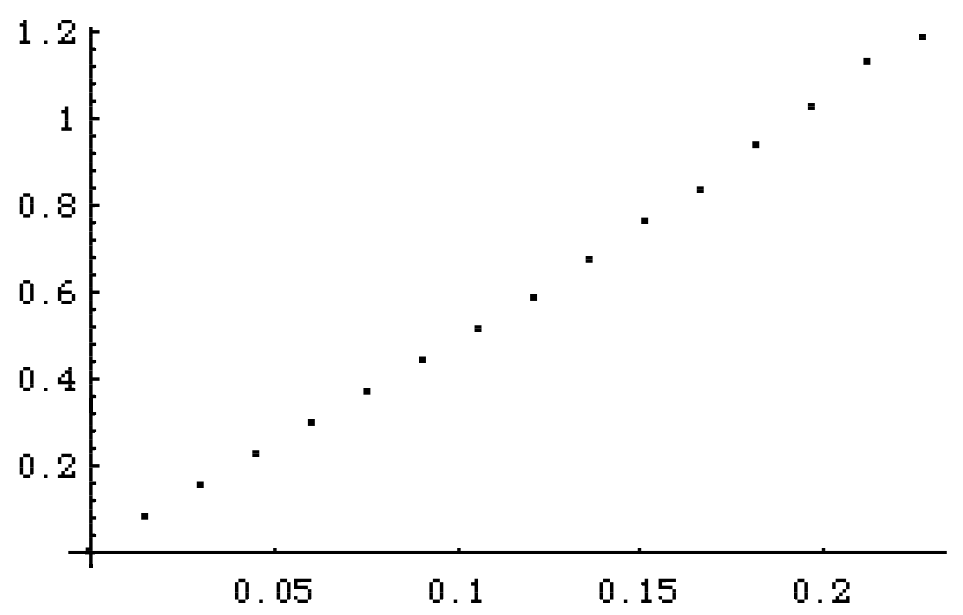

Fig. 5 Measured values of the position function versus normalized radius

For a better comparison Fig. 6 shows the experimental data plotted as points on the theoretical curve contained in Fig 2 above.

\section{Show[\%, p1, GridLines->Automatic]}

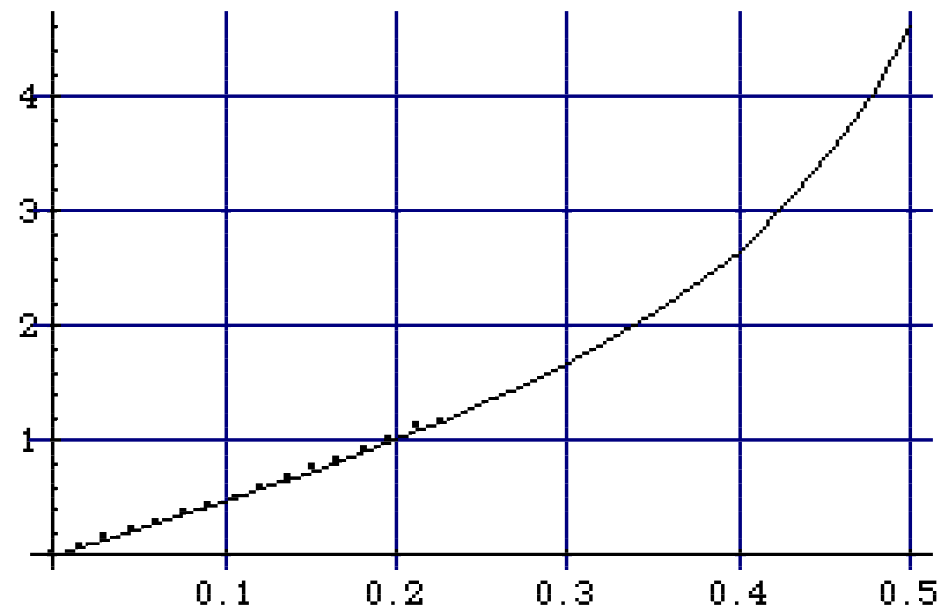

Fig 6. Comparison of the measured values with theory

\section{$\square$ References}


(1) Carrier, Krook, and Pearson, "Functions of a Complex Variable," McGraw-Hill Book Company, (1966), P. 129

(2) T.J. Fessenden, B.W. Stallard, and G.G. Berg, "Beam Current and Position Monitor for the Astron Accelerator", RSI. 43, p. 1789, (1972) 